\title{
Dynamical implications of multiple stellar populations
}

\author{
Alison I. Sills and Jonathan M. Downing \\ Department of Physics and Astronomy, McMaster University, \\ 1280 Main Street West, Hamilton, ON, L8S 4M1, Canada \\ email: asills@mcmaster.ca
}

\begin{abstract}
We investigate some implications of having two star formation episodes in globular clusters, rather than the traditional single-burst approximation. Evidence for more than one stellar generation is accumulating in observations of abundances of elements lighter than iron in globular cluster stars, and is thought to imply some self-enrichment of the globular cluster gas. In particular, we explore models based on the assumption that the self-enrichment comes from an early generation of asymptotic giant branch (AGB) stars.
\end{abstract}

Keywords. stars: AGB and post-AGB, globular clusters: general, globular clusters: individual (NGC 2808)

\section{Introduction}

Globular clusters are usually thought of as a simple population of stars that were formed in a single burst from a cloud of uniform composition. However, such a model is inconsistent with the detailed observations of abundances in globular cluster stars. Almost all clusters seem to have a constant value of iron or iron peak elements in their stars. However, every cluster that has been carefully studied shows star-to-star variation of lighter metals (C, N, O, Al, Mg, etc.) and possibly also helium for stars in all regions of the HR diagram. One possible mechanism to explain these variation is the self-enrichment scenario. This scenario takes a variety of forms, but always involves more than one star formation episode in the cluster. Dynamical models of star clusters have, to date, always assumed that reasonable 'initial conditions' are a coeval population of stars in dynamical equilibrium. These models tend to reproduce cluster observations well, but are inconsistent with the self-enrichment scenario. In this paper, we look at the dynamical implication of a second stellar generation. We follow other work (e.g., D'Antona \& Caloi 2004) in assuming that the first generation is weighted towards stars which have a significant AGB phase.

\section{Dynamical models}

We use STARLAB (Portegies Zwart et al. 2001) and a micro-GRAPE (Fukushige et al. $2005)$ to perform a direct $N$-body integration of our two-generation cluster. Our initial conditions consist of a Plummer distribution of the first generation of stars, which has an initial mass function (IMF) that is weighted such that there is an over-abundance of $3-5 \mathrm{M}_{\odot}$ stars. These are stars which will reach the AGB phase in about $200 \mathrm{Myr}$, and are the ones which produce the helium-enhanced material from which the second generation forms. We used the mass function from D'Antona \& Caloi (2004) which produced the smallest total mass of the cluster. This initial cluster was placed inside a static, analytic Plummer potential which represents the gas which will form the second generation. The mass of this potential was scaled so that it matched the mass lost from the first generation 


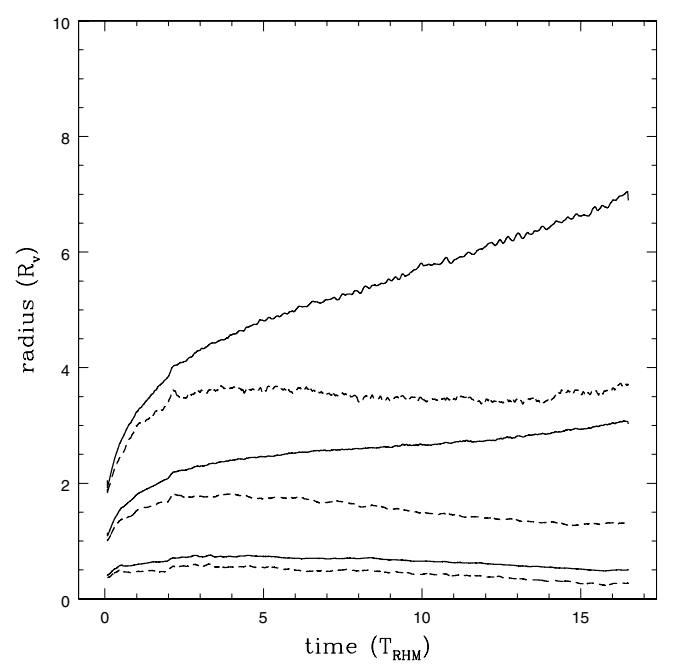

Figure 1. Time evolution of the $10 \%, 50 \%$ and $75 \%$ Lagrangian radii for a two-generation cluster, starting after the initial $200 \mathrm{Myr}$ evolution. The solid line shows the Lagrangian radii for all the stars in the cluster; the dotted line shows only the $10 \%$ most massive cluster members.

in 200 Myr. For simplicity, we assumed that there were no primordial binaries, there is no initial anisotropy, and that the cluster was not affected by the Galactic tidal field. Our initial virial radius for the cluster was taken to be $5 \mathrm{pc}$.

This initial cluster was evolved for $200 \mathrm{Myr}$. The simulation was then stopped. The Plummer potential representing the gas was removed, and a second generation of stars was added. This second generation had a Salpeter IMF, and was initially distributed in a Plummer sphere with the same scale radius as the first. The simulation was then restarted with the two generations, and allowed to evolve for at least $12 \mathrm{Gyr}$. Figure 1 shows the evolution of the $10 \%, 50 \%$ and $75 \%$ Lagrangian radii as a function of initial half mass relaxation times in the cluster. It looks completely normal, and dynamically the same as a single generation cluster. The only observable difference between the single and multiple generation clusters is that the two-generation clusters are more abundant in white dwarfs than would be expected from a normal stellar population. The velocity dispersions of the main sequence stars are indistinguishable from those of a normal Salpeter IMF cluster, and the mass-to-light ratio is only slightly higher $(\sim 2$ instead of $\sim 1.5)$.

\section{Conclusions}

Dynamically, we find that clusters which have two rapid bursts of star formation are almost indistinguishable from the canonical single generation clusters. The possible observational hints of a second generation come from the overabundance of white dwarfs compared to main sequence stars, and from the mass-to-light ratio. For more details of these simulations, see Downing \& Sills (2007).

\section{References}

D'Antona, F., \& Caloi, V. 2004, ApJ, 611, 871

Downing, J. M. B., \& Sills, A. I. 2007, ApJ, 662, 341

Fukushige, T., Makino, J., \& Kawai, A. 2005, PASJ, 57, 1009

Portegies Zwart, S. F., McMillan, S. L. W., Hut, P., \& Makino, J. 2001, MNRAS, 321, 199 